\title{
Comparative Effectiveness Of Fluoroquinolone Antibiotic Use In Uncomplicated Acute Exacerbations Of COPD: A Multi-Cohort Study
}

This article was published in the following Dove Press journal: International Journal of Chronic Obstructive Pulmonary Disease

Pierre Ernst, (D) ${ }^{1}$ Matthew Dahl, (D) ${ }^{2}$ Dan Chateau, (iD) ${ }^{2}$ Nick Daneman, ${ }^{3-6}$ Jacqueline Quail, ${ }^{7,8}$ Ingrid S Sketris, ${ }^{9}$ Anat Fisher, (D) 10 Jianguo Zhang," Shawn Bugden 12,13

On behalf of the Canadian Network for Observational Drug Effect Studies (CNODES) Investigators

'Centre for Clinical Epidemiology, Lady Davis Research Institute, Jewish General Hospital, Montreal, Quebec, Canada; ${ }^{2}$ Manitoba Centre for Health Policy, Department of Community Health Sciences, Max Rady College of Medicine, Rady Faculty of Health Sciences, University of Manitoba, Winnipeg, Manitoba, Canada; ${ }^{3}$ Institute for Clinical Evaluative Sciences, Toronto, Ontario, Canada; ${ }^{4}$ Sunnybrook Research Institute, Toronto, Ontario, Canada; ${ }^{5}$ Division of Infectious Diseases, Sunnybrook Health Sciences Centre, Toronto, Ontario, Canada; ${ }^{6}$ Faculty of Medicine, University of Toronto, Toronto, Ontario, Canada; ${ }^{7}$ Health Quality Council, Saskatoon, Saskatchewan, Canada; ${ }^{8}$ Department of Community Health \& Epidemiology, College of Medicine, University of Saskatchewan, Saskatoon, Saskatchewan, Canada; ${ }^{9}$ College of Pharmacy, Dalhousie University, Halifax, Nova Scotia, Canada;

${ }^{10}$ Department of Anesthesiology,

Pharmacology and Therapeutics, University of British Columbia, Vancouver, British Columbia, Canada; "Department of Medicine, Cumming School of Medicine, University of Calgary, Calgary, Alberta, Canada; ${ }^{12}$ School of Pharmacy, Memorial University of Newfoundland, St John's, Newfoundland and Labrador, Canada; ${ }^{13}$ College of Pharmacy, Rady Faculty of Health Sciences, University of Manitoba, Winnipeg, Manitoba, Canada

Correspondence: Shawn Bugden School of Pharmacy, Memorial University of Newfoundland, 300 Prince Philip Drive, St John's, Newfoundland and Labrador AIB 3V6, Canada

Tel $+1-709-864-4400$

$\mathrm{Fax}+1-709-864-4819$

Email shawn.bugden@mun.ca
Purpose: Fluoroquinolone antibiotics are associated with rare, but severe adverse events. They are frequently used for the treatment of acute exacerbations of COPD (AECOPD). While their effectiveness in severe exacerbations requiring hospitalisation has been well documented, the potential benefit in the ambulatory setting is less clear, especially in uncomplicated patients with COPD.

Patients and characteristics: We carried out a retrospective cohort study using health care databases from six Canadian provinces in subjects visiting their physician for uncomplicated COPD. Subjects dispensed either a quinolone or other antibiotics were compared using inverse probability of treatment weights with high dimensional propensity scores on 30-day outcomes, including repeat visits, hospitalisation for AECOPD and subsequent antibiotic prescription. Results from each province were combined by random effects meta-analysis.

Results: We identified 286,866 AECOPD events among 203,642 unique individuals. The frequency of fluoroquinolone use, mostly levofloxacin and moxifloxacin, varied by province and ranged from $8 \%$ to $32 \%$ of AECOPD antibiotic prescriptions. The risk of a repeat ambulatory care visit was increased among patients who were dispensed a fluoroquinolone compared with other antibiotics (OR 1.32, 95\% CI 1.27-1.36). The risk of a hospitalisation for AECOPD was also higher with fluoroquinolones (OR 1.52, 95\% CI 1.33-1.74). There was no difference in subsequent antibiotic prescriptions (OR 1.00, 95\% CI 0.94-1.07).

Conclusion: There is no apparent benefit in short-term outcomes with fluoroquinolones as compared to other antibiotics for the ambulatory treatment of AECOPD in uncomplicated patients. These findings support current recommendations that fluoroquinolones be reserved for AECOPD in patients with recurrent exacerbations, significant co-morbidity or requiring hospitalisation.

Keywords: chronic obstructive pulmonary disease, fluoroquinolones, comparative effectiveness

\section{Introduction}

Chronic obstructive pulmonary disease (COPD) is an important cause of morbidity and mortality and has become the third leading cause of death in the world. ${ }^{1,2}$ Acute exacerbations are an important event in COPD with many therapies directed at their prevention $^{3}$ and these exacerbations impact significantly on patient-related outcomes. ${ }^{4}$ An US Food and Drug Administration (FDA) briefing document on the benefits and risks of systemic fluoroquinolones, based on review of 15 placebocontrolled trials of anti-bacterial treatment of acute exacerbations of COPD 
(AECOPD), found that evidence of benefit was inconsistent, especially for mild exacerbations. The benefit was more evident for severe exacerbations requiring hospitalisation. ${ }^{5}$ Similar results have been reported by the Cochrane Collaboration which found a strong benefit in intensive care unit patients, but low-quality evidence and modest benefit overall. $^{6}$ Guidelines suggest treating AECOPD with antibiotics for exacerbations of at least moderate disease severity, as indicated by at least two of increased sputum volume, purulence, or worsening dyspnea. ${ }^{1,7,8}$ First-line antibiotics suggested include macrolides, second- or third-generation cephalosporins, trimethoprim-sulfamethoxazole, or doxycycline. Respiratory fluoroquinolones are reserved for patients with severe COPD, significant cardiac co-morbidity or frequent exacerbations. ${ }^{1}$ The fluoroquinolones appear effective in treating COPD exacerbations even with short courses. $^{9}$ There was concern early that they might be overused with increasing microbial resistance. ${ }^{10}$ Concern as to overuse of fluoroquinolones increased in pertinence with the report of rare but potentially disabling adverse effects such as Achilles tendonitis with rupture, ${ }^{11}$ aortic aneurysm, ${ }^{12}$ retinal detachment ${ }^{13}$ and peripheral neuropathy. ${ }^{14}$ Widespread use of fluoroquinolones has continued despite these concerns. ${ }^{5}$ For example, fluoroquinolones were the second most widely prescribed class of antibiotics in subjects over the age of 65 years in Ontario, Canada from 2006 to $2015 .{ }^{15}$ Serious adverse effects continue to be reported leading to advisories from Health Canada $^{16}$ and the FDA, ${ }^{17}$ most recently for aortic aneurysms. The National Institute for Health and Care Excellence (NICE) from the UK has also recently suggested a more restricted use of antibiotics in AECOPD. ${ }^{18}$ We wished to examine, using administrative health care databases in Canada, whether the use of fluoroquinolones in patients with uncomplicated acute exacerbations of COPD is of benefit, thus justifying their use in this situation.

\section{Materials And Methods \\ Data Sources}

We conducted a retrospective cohort study to compare the effectiveness of fluoroquinolone versus non-fluoroquinolone antibiotics in the treatment of uncomplicated acute exacerbations of COPD. The study was conducted under the auspices of the Canadian Network for Observational Drug Effect Studies (CNODES), a distributed network of investigators and linked databases across the majority of Canadian provinces. ${ }^{19,20}$ In each province of the CNODES network, the universal healthcare system provides validated, population-wide, de-identified healthcare utilization data, linkable at the patient level using encoded healthcare numbers. Dispensed drug prescriptions are available for almost all subjects 65 years or older $(>97 \%$ for most provinces; Nova Scotia 69\%). A common protocol was implemented separately within each of the provinces and aggregate results were pooled across provinces. We used these administrative health databases from the provinces of Alberta, British Columbia, Manitoba, Nova Scotia, Ontario and Saskatchewan.

\section{Inclusion/Exclusion Criteria}

Subjects were included in the cohort if they had a physician or nurse practitioner visit with a diagnostic code for COPD (ICD-9 490.x, 491.x, 492.x, 496.x and ICD-10 J40.x-J44.x), were at least 66 years of age, and had health care coverage for a minimum of 365 days before the visit for COPD. COPD visits with a dispensing of an antibiotic within 5 days were considered to represent an AECOPD. To limit the cohort to uncomplicated acute exacerbations of COPD, we excluded subjects with a medical outpatient visit for COPD, a hospitalisation for any cause, or use of an antibiotic or oral corticosteroid in the prior 90 days. These exclusions, within the limitations of the administrative data, are consistent with guideline definitions of uncomplicated acute exacerbations of COPD. ${ }^{7}$ We also excluded subjects with ischemic heart disease or heart failure in the prior year. Subjects were also required to have at least 35 days of information after the AECOPD.

\section{Outcomes}

Outcomes examined were repeat ambulatory care visits for COPD, hospitalisation with COPD as a diagnostic code in any position, or subsequent antibiotic prescriptions, all within 30 days of the antibiotic dispensing date used to identify an AECOPD. The study period was variable between databases but information was collected between January 1st, 2005 and March 31st, 2017. The study protocol was approved by the institutional review boards at all participating sites.

For each outcome, marginal adjusted odds ratios (OR) and their 95\% confidence intervals (CI) were estimated using logistic regression adjusting for age and calendar year. High-dimensional propensity scores were 
computed to identify and control for confounders and inverse probability of treatment weights (IPTW) were used to ensure comparable treatment groups. ${ }^{21,22}$ Aggregate results within each province were then pooled across provinces, weighted by the inverse of the standard error of the estimate, using a random effects model. Heterogeneity across provinces was assessed using the $\mathrm{I}^{2}$ statistic. As sensitivity analyses, individual patients were considered only once with the AECOPD event chosen at random, or the observation period after the antibiotic was dispensed was increased from 30 days to 60 days.

\begin{tabular}{|}
\hline AECOPD diagnoses events between \\
January 1, 2005 and latest date of data \\
available for the site \\
$(n=12,166,600)$
\end{tabular}

Exclusions $(n=11,897,714)$

- $<66$ years on the AECOPD event date $(n=5,357,087)$

- $\quad$ AECOPD event in the prior 90 days ( $n=4,032,399$ )

- Hospitalisation in the prior 90 days $(n=302,034)$

- Antibiotic use in the prior 90 days ( $n=587,415)$

- Oral corticosteroid use in the prior 90 days ( $n=68,343$ )

- $\quad$ AECOPD event occurs within 35 days prior to latest date of data available for the site $(n=59,109)$

- $\quad<365$ days of health coverage prior to the AECOPD event date $(n=8,036)$

- History of heart failure or ischemic heart disease in the year prior to or on the AECOPD event date $(n=445,210)$

- $\quad$ No health coverage for at least 35 days after the AECOPD event date $(n=3,857)$

- No antibiotic treatment on or within 5 days after the AECOPD event date $(n=1,034,224)$

\section{Number of new AECOPD events \\ $(n=268,886)$ \\ Number of unique individuals

$$
(n=203,642)
$$

Figure I Flow chart for study cohort creation.

Note: Patients were allowed to enter the cohort multiple times with a new AECOPD event.

Abbreviation: AECOPD, acute exacerbation of chronic obstructive pulmonary disease. 


\section{Results}

We identified 268,886 AECOPD events among 203,642 unique individuals (Figure 1). Overall fluoroquinolones, mostly levofloxacin and moxifloxacin, were used $20.8 \%$ of the time but the use varied from $8 \%$ to $32 \%$ of AECOPD, depending on the province. The most commonly dispensed non-fluoroquinolone antibiotics were clarithromycin, azithromycin, amoxicillin, or doxycycline (Table 1). Higher crude outcome event rates for repeat ambulatory care visits, hospitalisations, and subsequent antibiotic prescriptions were observed among patients treated with a fluoroquinolone compared to those treated with other antibiotics (Table 2). Propensity weighted analyses are provided by Forest plots in Figures 2-4. Figure 2 provides the risk of a repeat ambulatory care visit. Overall this is increased among patients dispensed a fluoroquinolone (OR 1.32, 95\% CI 1.27-1.36). Figure 3 shows that the risk of hospitalisation within 30 days of being dispensed a fluoroquinolone is higher than for subjects prescribed other antibiotics (OR 1.52, 95\% CI 1.33-1.74). There was no difference in subsequent antibiotic prescriptions

Table I Frequency Of Antibiotics Used For Initial Treatment Of Acute Exacerbations Of COPD

\begin{tabular}{|l|l|l|}
\hline Antibiotic & Count & Percent \\
\hline Clarithromycin & 54,292 & $20.2 \%$ \\
Azithromycin & 48,992 & $18.2 \%$ \\
Amoxicillin & 38,376 & $14.3 \%$ \\
Moxifloxacin & 27,829 & $10.3 \%$ \\
Doxycycline & 21,749 & $8.1 \%$ \\
Levofloxacin & 19,254 & $7.2 \%$ \\
Cefuroxime & 14,971 & $5.6 \%$ \\
Amoxicillin/Clavulanate & 10,366 & $3.9 \%$ \\
Ciprofloxacin & 8088 & $3.0 \%$ \\
Cephalexin & 6975 & $2.6 \%$ \\
\hline
\end{tabular}

(OR 1.00, 95\% CI 0.94-1.07) (Figure 4). Results were very similar when considering only one AECOPD per individual or when extending the period of observation to 60 days after the antibiotic was dispensed (data not shown).

\section{Discussion}

We identified more than 200,000 subjects receiving an antibiotic concomitantly with a physician visit for COPD. We then compared short-term outcomes between subjects dispensed a fluoroquinolone versus another antibiotic after weighting on the propensity of receiving a fluoroquinolone rather than another antibiotic in an attempt to assure that subjects were comparable. Subjects dispensed a fluoroquinolone had more repeat physician visits and greater risk of hospitalisation within 30 days than those prescribed other antibiotics. The need for a subsequent antibiotic dispensing (within 30 or 60 days) was the same.

A recent FDA study examined utilization data but not outcomes using administrative and physician survey data. ${ }^{5}$ They found fluoroquinolones were associated with acute exacerbations of COPD 59.4\% of the time in 2010 and $36.5 \%$ in 2014. While these values are higher than the $20.4 \%$ fluoroquinolone use found in this study, our approach targeted only uncomplicated exacerbations of COPD. A small study of primary care practice in the Netherlands evaluated the antibiotic prescribing of 1297 patients with exacerbations of COPD. ${ }^{23}$ Only $2 \%$ of the patients with single exacerbations received fluoroquinolone prescriptions. There was considerable variability across provinces in the rate of fluoroquinolone prescribing $(8-32 \%)$ but even the lowest rate exceeds that seen in the Netherlands. This variability in the quality of prescribing for exacerbations across jurisdictions has been seen in other studies but the root causes of these differences require further investigation. ${ }^{24}$

Table 2 Crude Outcome Event Rates (\%) Among AECOPD Treated With A Fluoroquinolone Compared With Other Antibiotics

\begin{tabular}{|l|l|l|l|l|l|l|}
\hline \multirow{2}{*}{ Province } & \multicolumn{2}{l|}{$\begin{array}{l}\text { Repeat Ambulatory Care Visit For } \\
\text { AECOPD }\end{array}$} & \multicolumn{2}{l|}{$\begin{array}{l}\text { Hospitalisation For } \\
\text { AECOPD }\end{array}$} & \multicolumn{2}{l|}{$\begin{array}{l}\text { Subsequent Antibiotic } \\
\text { Dispensation }\end{array}$} \\
\cline { 2 - 7 } & FQ & Other AB & FQ & Other AB & FQ & Other AB \\
\hline Alberta & 24.5 & 17.7 & 3.25 & 1.79 & 16.8 & 16.3 \\
British Columbia & 26.0 & 19.8 & 1.63 & 0.65 & 16.8 & 16.1 \\
Manitoba & 23.8 & 15.9 & 2.05 & 0.91 & 17.0 & 15.1 \\
Nova Scotia & 30.9 & 22.1 & 2.47 & 1.22 & 17.7 & 15.1 \\
Ontario & 22.7 & 17.4 & 3.23 & 1.98 & 16.7 & 16.7 \\
Saskatchewan & 27.3 & 17.2 & 4.43 & 1.35 & 19.1 & 16.1 \\
\hline
\end{tabular}

Abbreviations: $A B$, antibiotics; $A E C O P D$, acute exacerbation of chronic obstructive pulmonary disease; FQ, fluoroquinolones. 


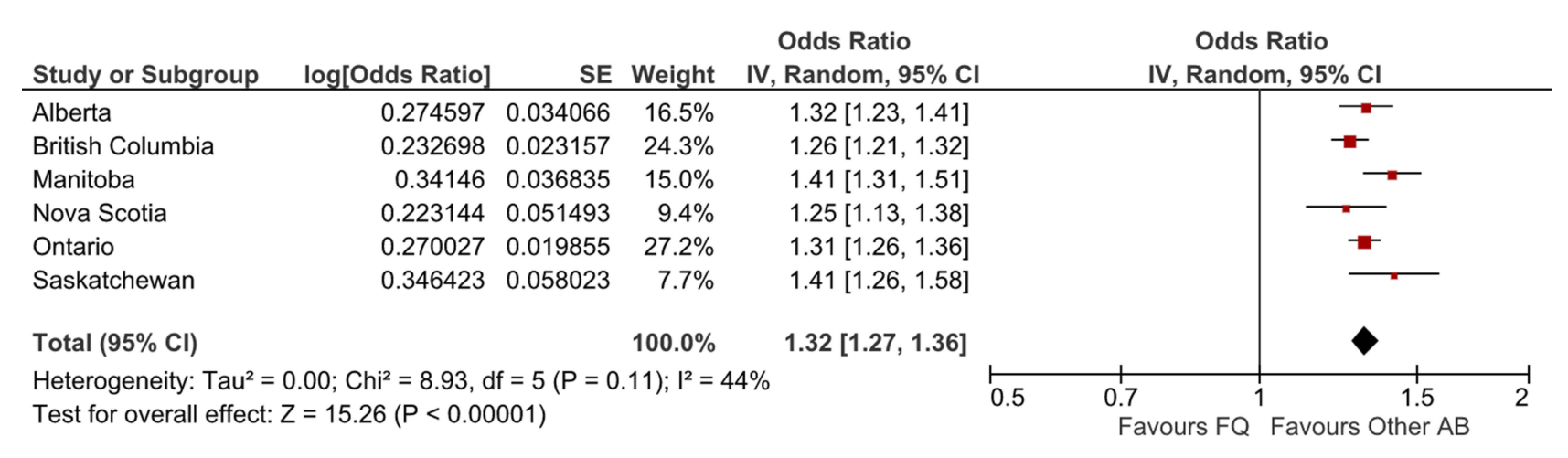

Figure 2 ORs $(95 \% \mathrm{Cl})$ for repeat ambulatory care visits associated with AECOPD initially treated with a fluoroquinolone compared with other antibiotics. Abbreviations: $A B$, antibiotics; $A E C O P D$, acute exacerbation of chronic obstructive pulmonary disease; $C l$, confidence interval; $F Q$, fluoroquinolones; OR, odds ratio; $S E$, standard error; IV, inverse variance.

\begin{tabular}{|c|c|c|c|c|c|c|c|c|}
\hline Study or Subgroup & log[Odds Ratio] & SE & Weight & $\begin{array}{c}\text { Odds Ratio } \\
\text { IV, Random, } 95 \% \mathrm{CI}\end{array}$ & \multicolumn{4}{|c|}{$\begin{array}{c}\text { Odds Ratio } \\
\text { IV, Random, } 95 \% \mathrm{Cl}\end{array}$} \\
\hline Alberta & 0.330742 & 0.089515 & $19.2 \%$ & $1.39[1.17,1.66]$ & & & & \\
\hline British Columbia & 0.534737 & 0.093201 & $18.7 \%$ & $1.71[1.42,2.05]$ & & & & \\
\hline Manitoba & 0.276874 & 0.12966 & $14.0 \%$ & $1.32[1.02,1.70]$ & & & & \\
\hline Nova Scotia & 0.336472 & 0.166593 & $10.5 \%$ & $1.40[1.01,1.94]$ & & & & \\
\hline Ontario & 0.329304 & 0.050098 & $25.1 \%$ & $1.39[1.26,1.53]$ & & & & \\
\hline Saskatchewan & 0.801104 & 0.14434 & $12.5 \%$ & $2.23[1.68,2.96]$ & & & & \\
\hline Total $(95 \% \mathrm{Cl})$ & & & $100.0 \%$ & $1.52[1.33,1.74]$ & & & & \\
\hline \multicolumn{9}{|c|}{ 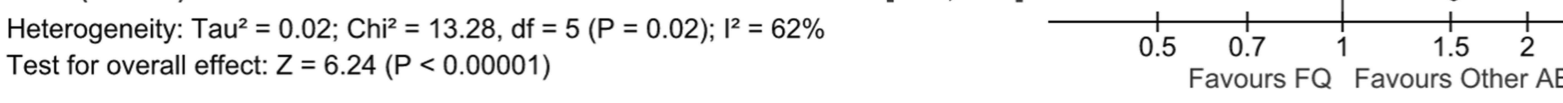 } \\
\hline
\end{tabular}

Figure 3 ORs $(95 \% \mathrm{Cl})$ for hospitalisations associated with AECOPD initially treated with a fluoroquinolone compared with other antibiotics.

Abbreviations: $\mathrm{AB}$, antibiotics; $\mathrm{AECOPD}$, acute exacerbation of chronic obstructive pulmonary disease; $\mathrm{Cl}$, confidence interval; $\mathrm{FQ}$, fluoroquinolones; OR, odds ratio; SE, standard error; IV, inverse variance.

\begin{tabular}{|c|c|c|c|c|c|c|c|}
\hline Study or Subgroup & log[Odds Ratio] & SE & Weight & $\begin{array}{c}\text { Odds Ratio } \\
\text { IV, Random, } 95 \% \mathrm{Cl}\end{array}$ & & $\begin{array}{c}\text { Odds Ratio } \\
\text { IV, Random, } 95 \% \mathrm{CI}\end{array}$ & \\
\hline Alberta & 0.023717 & 0.037197 & $17.4 \%$ & $1.02[0.95,1.10]$ & & & \\
\hline British Columbia & -0.04082 & 0.02729 & $19.2 \%$ & $0.96[0.91,1.01]$ & & & \\
\hline Manitoba & 0.014889 & 0.041341 & $16.6 \%$ & $1.02[0.94,1.10]$ & & & \\
\hline Nova Scotia & -0.00401 & 0.057411 & $13.5 \%$ & $1.00[0.89,1.11]$ & & & \\
\hline Ontario & -0.09431 & 0.017103 & $20.7 \%$ & $0.91[0.88,0.94]$ & & - & \\
\hline Saskatchewan & 0.191446 & 0.062204 & $12.7 \%$ & $1.21[1.07,1.37]$ & & & \\
\hline Total $(95 \% \mathrm{Cl})$ & & & $100.0 \%$ & $1.00[0.94,1.07]$ & & & \\
\hline \multicolumn{8}{|c|}{ 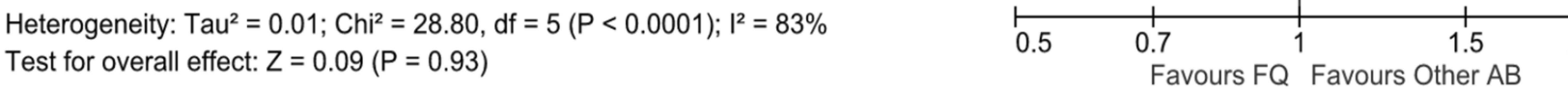 } \\
\hline
\end{tabular}

Figure 4 ORs $(95 \% \mathrm{Cl})$ for subsequent antibiotic dispensations associated with AECOPD initially treated with a fluoroquinolone compared with other antibiotics. Abbreviations: $A B$, antibiotics; $A E C O P D$, acute exacerbation of chronic obstructive pulmonary disease; Cl, confidence interval; $F Q$, fluoroquinolones; OR, odds ratio; $\mathrm{SE}$, standard error; IV, inverse variance.

This population-based study of multiple cohorts has several strengths. The population and number of events are large permitting precise estimates in a real-world setting. Results were consistent in each of the provinces assuring that the results are generalisable. We used stateof-the-art methods to adjust for potential confounding. Of note, the results only apply to uncomplicated AECOPD, since we deliberately excluded those with recent 
exacerbations, hospitalisation, or significant cardiac comorbidity as 2nd line antibiotics like fluoroquinolones may be justified in these patients.

This study has several limitations. First, the diagnosis of COPD is based on outpatient physician billing codes which may not be accurate since we do not have access to lung function test results. We assumed that a visit for COPD linked to a prescription for a respiratory antibiotic was indicative of a COPD exacerbation. This may, however, represent the dispensing of an antibiotic as part of an action plan for a future exacerbation. We do not think that this would be a common occurrence in the relatively uncomplicated patients with COPD we selected. Also, the antibiotic may have been prescribed for a concomitant pneumonia or other infection. We cannot be sure the weighting of patients by propensity scores is sufficient to eliminate confounding. We did not have important information such as lung function, use of home oxygen, smoking status, symptom scores or colonisation with more resistant bacteria such as Pseudomonas, that might have allowed us to render the treatment groups more comparable and help further ensure that more complicated exacerbations were excluded. Therefore, we do not feel that the poorer outcomes among those prescribed fluoroquinolones are causal but more likely reflect a more severe population.

In conclusion, treatment of apparently uncomplicated COPD exacerbations with fluoroquinolone versus nonfluoroquinolone antibiotics does not appear to be associated with improved outcomes in the following 30 days, including repeat ambulatory care visits, hospitalisation for COPD or repeat antibiotic prescriptions. These findings support current recommendations to limit the use of fluoroquinolones to more complicated patients with recurrent exacerbations or significant cardiac co-morbidity. Such judicious use is especially important given the rare but severe adverse effects associated with the use of fluoroquinolone antibiotics.

\section{Acknowledgments}

We would like to acknowledge the programming support of Zhihai Ma (Alberta), Hala Tamim, Yan Wang and Steve Doucette (Nova Scotia), Fangyun Wu and Vicky Ling (Ontario), and Nianping Hu (Saskatchewan). We would like to acknowledge the important contributions of the Canadian Network for Observational Drug Effect Studies (CNODES) collaborators and assistants at each site. The CNODES investigators are: Samy Suissa (Principal Investigator); Colin R. Dormuth (British Columbia); Brenda R. Hemmelgarn
(Alberta); Gary F. Teare and Jacqueline Quail (Saskatchewan); Patricia Caetano and Dan Chateau (Manitoba); David A. Henry and J. Michael Paterson (Ontario); Jacques LeLorier (Québec); Adrian R. Levy (Atlantic: Nova Scotia, Newfoundland and Labrador, New Brunswick, Prince Edward Island); Pierre Ernst and Kristian B. Filion (UK Clinical Practice Research Datalink (CPRD)); Robert W. Platt (Methods); and Ingrid S. Sketris (Knowledge Translation). CNODES, a collaborating centre of the Drug Safety and Effectiveness Network (DSEN), is funded by the Canadian Institutes of Health Research (Grant Number DSE146021).

This study was made possible through data-sharing agreements between CNODES member research centres and the respective provincial governments of Alberta, British Columbia, Manitoba (HIPC \#2016/2017 - 15), Nova Scotia, Ontario, and Saskatchewan. The BC Ministry of Health approved access to and use of $\mathrm{BC}$ data facilitated by Population Data BC for this study. British Columbia data sources were as follows (http:// www.popdata.bc.ca/data): British Columbia Ministry of Health [creator] (2018): Medical Services Plan (MSP) Payment Information File. BC Ministry of Health [publisher]. MOH (2018); British Columbia Ministry of Health [creator] (2018): PharmaNet. BC Ministry of Health [publisher]. Data Stewardship Committee (2018); Canadian Institute for Health Information [creator] (2018): Discharge Abstract Database (Hospital Separations). BC Ministry of Health [publisher]. MOH (2018); British Columbia Ministry of Health [creator] (2018): Consolidation File (MSP Registration \& Premium Billing). BC Ministry of Health [publisher]. $\mathrm{MOH}$ (2018). All inferences, opinions, and conclusions drawn in this manuscript are those of the authors, and do not reflect the opinions or policies of the Data Steward(s). Parts of this material are based on data and/or information compiled and provided by the Canadian Institute for Health information (CIHI). The opinions, results and conclusions reported in this paper are those of the authors and are independent from the funding sources. No endorsement by the provinces, data stewards, or CIHI is intended or should be inferred.

\section{Author Contributions}

All authors contributed to the data analysis, drafting and revising the article, gave final approval to the published version, and agree to be accountable for all aspects of the work. 


\section{Disclosure}

Dr Ingrid Sketris reports salary support in part from CIHR for the CNODES project, during the conduct of the study, grants from Nova Scotia Government's Drug Evaluation Alliance of Nova Scotia, CIHR, and Australian NHMRC, outside the submitted work. The authors report no other conflicts of interest in this work.

\section{References}

1. Vogelmeier CF, Criner GJ, Martinez FJ, et al. Global strategy for the diagnosis, management, and prevention of chronic obstructive lung disease 2017 report. GOLD executive summary. Am J Respir Crit Care Med. 2017;195(5):557-582. doi:10.1164/rccm.201701-0218PP

2. GBD. 2015 mortality and causes of death collaborators. Global, regional, and national life expectancy, all-cause mortality, and cause-specific mortality for 249 causes of death, 1980-2015: a systematic analysis for the Global Burden of Disease Study 2015. Lancet. 2016;388 (10053):1459-1544

3. Wedzicha JA, Calverley PM, Seemungal TA, Hagan G, Ansari Z, Stockley RA. The prevention of COPD exacerbations by salmeterol/ fluticasone propionate or tiotropium bromide. Am J Respir Crit Care Med. 2008;177(1):19-26. doi:10.1164/rccm.200707-973OC

4. Seemungal TAR, Donaldson GC, Paul EA, Bestall JC, Jeffries DJ, Wedzicha JA. Effect of exacerbation on quality of life in patients with chronic obstructive pulmonary disease. Am J Respir Crit Care Med. 1998;157:1418-1422. doi:10.1164/ajrccm.157.5.9709032

5. FDA Briefing Document; Joint Meeting of the Antimicrobial Drugs Advisory Committee and the Drug Safety and Risk Management Advisory Committee. The benefits and risks of systemic fluoroquinolone antibacterial drugs for the treatment of Acute Bacterial Sinusitis (ABS), Acute Bacterial Exacerbation of Chronic Bronchitis in Patients Who Have Chronic Obstructive Pulmonary Disease (ABECB-COPD), and Uncomplicated Urinary Tract Infections (uUTI). 2015; p.1-42. Available from: https://www.pharmamedtechbi.com/ /media/ Supporting\%20Documents/The\%20Pink\%20Sheet\%20DAILY/2015/ November/Antimicrobial\%20AC\%20FDA\%20briefing\%2011515.pdf. Accessed January 11, 2019.

6. Vollenweider DJ, Frei A, Steurer-Stey CA, Garcia-Aymerich J, Puhan MA. Antibiotics for exacerbations of chronic obstructive pulmonary disease. Cochrane Database Syst Rev. 2018;10:Cd010257.

7. O'Donnell DE, Hernandez P, Kaplan A, et al. Canadian Thoracic Society recommendations for management of chronic obstructive pulmonary disease - 2008 update - highlights for primary care. Can Respir J. 2008;15(Suppl A):1a-8a. doi:10.1155/2008/641965

8. Laue J, Reierth E, Melbye H. When should acute exacerbations of COPD be treated with systemic corticosteroids and antibiotics in primary care: a systematic review of current COPD guidelines. NPJ Prim Care Respir Med. 2015;25:15002. doi:10.1038/ npjpcrm.2015.2

9. Anzueto A, Miravitlles M. Short-course fluoroquinolone therapy in exacerbations of chronic bronchitis and COPD. Respir Med. 2010;104 (10):1396-1403. doi:10.1016/j.rmed.2010.05.018
10. Williams JH Jr. Fluoroquinolones for respiratory infections: too valuable to overuse. Chest. 2001;120(6):1771-1775. doi:10.1378/ chest.120.6.1771

11. Daneman N, Lu H, Redelmeier DA. Fluoroquinolones and collagen associated severe adverse events: a longitudinal cohort study. BMJ Open. 2015;5(11):e010077. doi:10.1136/bmjopen-2015-010077

12. Pasternak B, Inghammar M, Svanstrom H. Fluoroquinolone use and risk of aortic aneurysm and dissection: nationwide cohort study. $B M J$. 2018;360:k678. doi:10.1136/bmj.k678

13. Etminan M, Forooghian F, Brophy JM, Bird ST, Maberley D. Oral fluoroquinolones and the risk of retinal detachment. JAMA. 2012;307 (13):1414-1419. doi:10.1001/jama.2012.383

14. Etminan M, Brophy JM, Samii A. Oral fluoroquinolone use and risk of peripheral neuropathy: a pharmacoepidemiologic study. Neurology. 2014;83(14):1261-1263. doi:10.1212/WNL.0000000000000846

15. Tan C, Graves E, Lu H, et al. A decade of outpatient antimicrobial use in older adults in Ontario: a descriptive study. CMAJ Open. 2017;5(4):E878-e885. doi:10.9778/cmajo.20170100

16. Health Canada. Fluoroquinolones - risk of disabling and persistent serious adverse reactions. Available from: http://healthycanadians. gc.ca/recall-alert-rappel-avis/hc-sc/2017/61900a-eng.php. Accessed November 18, 2018.

17. Food and Drug Administration. Fluoroquinolone antibiotics: safety communication - increased risk of ruptures or tears in the aorta blood vessel in certain patients. Available from: https://www.fda.gov/Safety/ MedWatch/SafetyInformation/SafetyAlertsforHumanMedicalProducts/ ucm628960.htm. Accessed January 11, 2019.

18. National Intitute for Health and Care Excellence (NICE) Guideline. Chronic obstructive pulmonary disease (acute exacerbation): antimicrobial prescribing. December. 2018;5:2018.

19. Suissa S, Henry D, Caetano P, et al. CNODES: the Canadian network for observational drug effect studies. Open Med. 2012;6(4): e134-140

20. Platt RW, Platt R, Brown JS, Henry DA, Klungel OH, Suissa S. How pharmacoepidemiology networks can manage distributed analyses to improve replicability and transparency and minimize bias. Pharmacoepidemiol Drug Saf. 2019. doi:10.1002/pds.4722

21. Curtis LH, Hammill BG, Eisenstein EL, Kramer JM, Anstrom KJ. Using inverse probability-weighted estimators in comparative effectiveness analyses with observational databases. Med Care. 2007;45(10 Supl 2):S103-107. doi:10.1097/MLR.0b013e318065 $18 \mathrm{ac}$

22. Schneeweiss S, Rassen JA, Glynn RJ, Avorn J, Mogun H, Brookhart MA. High-dimensional propensity score adjustment in studies of treatment effects using health care claims data. Epidemiology. 2009;20(4):512-522. doi:10.1097/EDE.0b013e3181a663cc

23. Bathoorn E, Groenhof HR, van der Molen T, et al. Real-life data on antibiotic prescription and sputum culture diagnostics in acute exacerbations of COPD in primary care. Int J COPD. 2017;12:285290. doi:10.2147/COPD.S120510

24. Lopez-Campos JL, Harti S, Pozo-Rodriguez F, Roberts CM. Antibiotic prescription for COPD exacerbations admitted to hospital: European COPD audit. PlosOne. 2015;10(4):e0123274. doi:10.1371/ journal.pone.0124374 


\section{Publish your work in this journal}

The International Journal of COPD is an international, peer-reviewed journal of therapeutics and pharmacology focusing on concise rapid reporting of clinical studies and reviews in COPD. Special focus is given to the pathophysiological processes underlying the disease, intervention programs, patient focused education, and self management protocols. This journal is indexed on PubMed Central, MedLine and CAS. The manuscript management system is completely online and includes a very quick and fair peer-review system, which is all easy to use. Visit http://www.dovepress.com/testimonials.php to read real quotes from published authors.

Submit your manuscript here: https://www.dovepress.com/international-journal-of-chronic-obstructive-pulmonary-disease-journal 